http://jmscr.igmpublication.org/home/ ISSN (e)-2347-176x ISSN (p) 2455-0450

crossref DOI: https://dx.doi.org/10.18535/jmscr/v7i11.117

\title{
Study of orbital fractures and ocular complications in road traffic accidents in two wheeler drivers
}

\author{
Authors \\ Dr Mahesh S.G ${ }^{1}$, Dr Manjula Y.M ${ }^{2 *}$
}

${ }^{1}$ Associate Professor, Department of Plastic Surgery, Kempegowda Institute of Medical Sciences, Bangalore

${ }^{2}$ Associate Professor, Department of Ophthalmology, BGS Global Institute of Medical Sciences, Bangalore *Corresponding Author

Dr Manjula Y.M

\begin{abstract}
Aim: Case study of 25 patients to understand the nature and type of orbital fractures and ocular complication in two wheeler drivers in road traffic accidents.

Methods: prospective study of 25 patients aged between 20-40years with history of road traffic accidents while driving two wheeler. Study was conducted over a period of one year from august 2018 to august2019. patients with only orbital fractures were included in the study.

Detailed history, clinical examination of face, eye, and orbit was done .visual acuity was recorded, investigation like X-ray orbit and PNS, CT scan was taken at time of presentation and CT scan was repeated after 1month and 6month after the management of cases. Patients with Soft tissue injury without orbital fractures were managed medically by oral antibiotic and NSAIDS. Local moxifloxacin $0.5 \%$ eye ointment was used for skin abrasion and lacerated sutured wound.

Displaced comminuted lateral wall (zygoma) fractures were managed by open reduction with interfragment wire fixation or plate and screw fixation (ORIF). Orbital floor reconstruction was done using titanium mesh. All patients had regular follow up at 1week, 1month, 3month and 6month after the management of cases.

Result: All the patient in our study fell in age group of 20-40years .with male preponderance, 19 were male patients. Commonest nature of injury was by direct collision of two wheeler with another vehicle.

$24 \%(6)$ of patient had isolated lateral wall fracture, 8\%(2) had isolated medial wall fracture, 8\%(2) had isolated floor fracture, lateral wall fracture combined with other wall fracture was maximum 60\%(15).

All patients had periorbital ecchymosis, and subconjunctival hemorrhage. Lid laceration was noted in 10 patients were suturing was done with 8-0 silk.

Extra ocular muscle movement restriction was noted in 8 patients. Enophthalmos, diplopia and infraorbital anesthesia was noted in 2 patients who had severe orbital floor fracture which was corrected by surgery. 2 patient developed traumatic cataract after 2months for which small incision cataract surgery with posterior chamber intraocular lens implantation was done. 4 patients had vitreous hemorrhage which was managed medically.

In our study $80 \%$ of the patient had normal vision only $20 \%$ had decreased vision. No one was blind.

Conclusion: In our study multiple orbital wall fracture was common 60\%, followed by isolated lateral wall fracture 24\%. lateral orbital wall fracture are most common in two wheeler drivers following road traffic accidents because lateral orbital wall being most prominent orbital structure susceptible to direct impact after fall from two wheeler. Multiple orbital wall fracture was common in two wheeler driver without helmet.

Keywords: orbital wall fractures, maxillofacial injuries, ocular complications, road traffic accidents, two wheeler drivers, ORIF-open reduction with internal fixation.
\end{abstract}




\section{Introduction}

Injuries of the maxillofacial skeleton are extremely frequent in modern era, their incidence having increased over past decade. Being the most exposed part of the body, the face is particularly vulnerable to injuries.

Ocular trauma is a major cause of worldwide visual impairment. Doctors are exposed to selected aspect of ocular trauma, depending up on specific type of practice.

The etiology related to orbital and maxillofacial fractures are several varies from one country to another country and even within the same country depending on the prevailing socioeconomic, culture and environmental factors ${ }^{(1,2)}$, Second and third decades men are mainly engaged in activities that can predispose them to trauma and maxillofacial fractures. ${ }^{(1,-7)}$.

Ocular injuries can and do occur in many circumstances like sports activity, recreation, workplace, home, rural agricultural setting, assault and motor vehicle accidents. With increase in vehicles maxillofacial fractures and ocular injury due to road traffic accidents has increased. This observation is also agreement with the studies by Guven $^{(1)}$, Tanaka et $\mathrm{al}^{(11)}$ and Moshy et $\mathrm{al}^{(12)}$.

Ocular injuries Varying from simple undisplaced hair line fracture of orbital walls to gross displaced fracture of orbital walls and trauma of the face, management of those diseases can be challenging for surgeons and can have effect on psychosocial and functional activities of the patient ${ }^{(1,2,4)}$.The mid facial fractures can involve both hard and soft tissue areas of the face, and sometimes, they can be related with other systemic pathologies and requiring multidisciplinary management There should be protocol for management of airways ,breathing, and circulation. The orbital trauma is the hardest to be treated for its location and related signs.

The management strategies in different set-up also vary according to the protocol, the infrastructure and technical expertise of the treating surgeons.

The oldest report of fracture Zygoma and management are reported in Smith papyrus ${ }^{(8)}$.
Lang was first to describe the so-called "blowout" fracture of the floor of orbit ${ }^{(9)}$. Treatment of orbital injuries varies from medical management or surgery. Orbital wall fractures were treated by closed reduction if it is non comminuted undisplaced. Displaced comminuted fractures mainly zygomatic fractures were treated with open reduction using wires fixation, miniplate and screws.

Depressed (blow -out) fracture of the orbital floor may be difficult to discover because of the presence of orbital and periorbital edema, haematoma. Presence of enophthalmos, diplopia herniation of orbital content to maxillary sinus was treated by open fracture reduction and reconstruction of orbital floor by using collagen, bony graft or titanium mesh ${ }^{(10)}$.

\section{Material and Methods}

Our study was prospective study of 25 patients who were aged between 20-40 years with history of road traffic accidents while driving two wheeler with only orbital fracture were included in the study.

The study was conducted over a period of one year from august 2018 to august2019

Informed consent was obtained from all the patients.

Detailed history about injury was taken.

Clinical examination of face, Eye (anterior segment of eye examination was done using slit lamp and posterior segment eye examination was done after dilation of pupil after checking pupillary reaction using indirect ophthalmoscope) Visual acuity was tested using snellens chart, at the time of presentation and in every follow up.

Orbital margins were palpated for any crepitus and margin irregularity.

Examination of all patients was done by plastic surgeon and ophthalmologist.

Investigation - like X-ray orbit and PNS,

CT scan with coronal and sagittal scan was taken at time of presentation and repeated after 1month, and 6 month of management in all cases suspected with orbital fractures. 
Patients with severe mandibular and maxillary fracture along with orbital fracture, patients with head injury with bleeding in cranium were excluded from the study.

All patients had periorbital ecchymosis, and subconjunctival hemorrhage.

Lid laceration was noted in 10 patients that were sutured using with 8-0 silk.

Extra ocular muscle movement restriction was noted in 8 patients. After treatment all patient regained normal extraocular muscle movement at the end of follow up.

Enophthalmos diplopia and infraorbital anesthesia was noted in 2 patients who had severe orbital floor fracture which was corrected by surgery. Transconjunctival approach to orbital floor fracture was used, orbital floor reconstruction was done using titanium mesh. After releasing of entrapped ocular muscle ${ }^{(13)}$ and fracture reduction under general anesthesia.

2 patient developed traumatic cataract after 2 months for which small incision cataract surgery with posterior chamber intraocular lens implantation was done post op vision was 6/9-6/6.

4 patients had vitreous hemorrhage which was managed medically.

$24 \%$ (6)of patient had isolated lateral wall fracture, $8 \%$ (2) had isolated medial wall fracture, $8 \%(2)$ had isolated floor fracture, lateral wall fracture combined with other wall fracture was maximum $60 \%(15)$.

Patients with Soft tissue injury without orbital fractures were management medically by oral antibiotic tablet amoxicillin 500mg+clavulanic acid $125 \mathrm{mg}$ twice daily for five days with NSAIDS diclofenac 50mgwith serratiopeptidase $10 \mathrm{mg}$ twice daily for three days. Local moxifloxacin $0.5 \%$ eye ointment was used for skin abrasion and lacerated sutured wound.

Displaced comminuted lateral wall (zygoma) fractures was managed by open reduction once periorbital swelling decreased within 10 days with interfragment wire fixation or plate and screw fixation (ORIF), under general anesthesia using two incision one in lateral end of the brow and other at the inferior margin of the lower lid. Brow incision provides access to zygomatico frontal suture line, infraorbital incision permits exposure and inspection of infraorbital margin and floor of orbit.

All surgery patients was put on intravenous cephazolin 1g 30-60minutes before surgical procedure Continued cephazolin $1 \mathrm{~g}$ i.v. twice daily for next 3-5 days.

All patients follow up was done after 1 week, 1 month, 3month and 6month. Wound healing and fracture reduction was assessed by repeat $\mathrm{CT}$ at 1 month and 6months.

\section{Results}

In prospective study of 25 patients with history of road traffic accidents while driving two wheeler who fulfilled above criteria results were.

1. Age group: In our study orbital fracture was more common in 21-30years of age group. This included 16 patients $(64 \%)$ out of 25 patients.

\begin{tabular}{|l|c|c|}
\hline Age & No of patients & Percentage\% \\
\hline $11-20$ years & 1 & $4 \%$ \\
\hline $21-30$ years & 16 & $64 \%$ \\
\hline 31-40years & 8 & $32 \%$ \\
\hline
\end{tabular}

\section{Male to female ratio was19:6.}

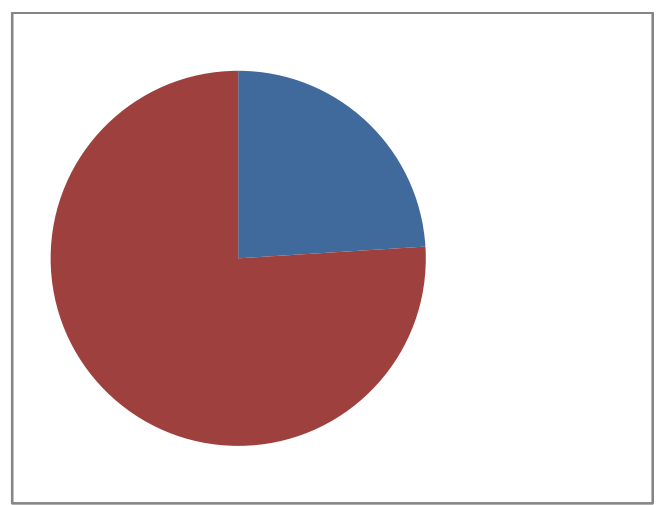

Majority of patients were males $19(76 \%)$ out of 25 in our study.

\section{Orbital wall fractures}

\begin{tabular}{|l|c|c|}
\hline Orbital wall fractured & $\begin{array}{c}\text { No of } \\
\text { patients }\end{array}$ & Percentage\% \\
\hline $\begin{array}{l}\text { Isolated lateral orbital } \\
\text { wall }\end{array}$ & 6 & $24 \%$ \\
\hline Isolated orbital floor & 2 & $8 \%$ \\
\hline Isolated medial wall & 2 & $8 \%$ \\
\hline Multiple orbital wall & 15 & $60 \%$ \\
\hline
\end{tabular}


In our study multiple orbital wall fracture was common $60 \%$, followed by isolated lateral wall fracture $24 \%$.

\section{Ocular complication}

\begin{tabular}{|l|c|c|}
\hline Ocular complications & $\begin{array}{c}\text { No of } \\
\text { patients }\end{array}$ & Percentage\% \\
\hline Periorbital ecchymosis & 25 & $100 \%$ \\
\hline Subconjunctival hemorrhage & 25 & $100 \%$ \\
\hline Lid laceration muscle & 10 & $40 \%$ \\
\hline $\begin{array}{l}\text { Extraocular } \\
\text { movement restriction }\end{array}$ & 2 & $32 \%$ \\
\hline enophthalmos & 2 & $8 \%$ \\
\hline diplopia & 2 & $8 \%$ \\
\hline Infraorbital anesthesia & 2 & $8 \%$ \\
\hline Traumatic cataract & 4 & $16 \%$ \\
\hline Vitreous hemorrhage & & \\
\hline
\end{tabular}

All patients had periorbital ecchymosis, and subconjunctival hemorrhage.

Lid laceration was noted in 10 patients that were sutured using with 8-0 silk.

Extra ocular muscle movement restriction was noted in 8 patients. After treatment all patient regained normal extraocular muscle movement at the end of follow up.

Enophthalmos, diplopia and infraorbital anesthesia was noted in 2 patients who had severe orbital floor fracture which was corrected by surgery. Transconjunctival approach to orbital floor fracture was used, orbital floor reconstruction was done using titanium mesh. After releasing of entrapped ocular muscle and fracture reduction.

2 patient developed traumatic cataract after 2 months for which small incision cataract surgery with posterior chamber intraocular lens implantation was done post op vision was 6/9-6/6.

4 patients had vitreous hemorrhage which was managed medically.

Traumatic optic neuropathy, retinal detachment, globe rupture, intraocular foreign body was not noted in any of the patient in our study.
$24 \%(6)$ of patient had isolated lateral wall fracture, $8 \%$ (2) had isolated medial wall fracture, $8 \%$ (2) had isolated floor fracture, lateral wall fracture combined with other wall fracture was maximum $60 \%$ (15).

Patients with Soft tissue injury without orbital fractures were management medically by oral antibiotic tablet amoxicillin 500mg+clavulanic acid $125 \mathrm{mg}$ twice daily for five days with NSAIDS diclofenac 50mgwith serratiopeptidase $10 \mathrm{mg}$ twice daily for three days. Local moxifloxacin $0.5 \%$ eye ointment was used for skin abrasion and lacerated sutured wound.

Displaced comminuted lateral wall (zygoma) fractures was managed by open reduction once periorbital swelling decreased within 10 days with interfragment wire fixation or plate and screw fixation, using two incision one in lateral end of the brow and other at the inferior margin of the lower lid. Brow incision provides access to zygomatico frontal suture line; infraorbital incision permits exposure and inspection of infraorbital margin and floor of orbit.

All surgery patients was put on intravenous cephazolin 1g 30-60minutes before surgical procedure Continued cephazolin $1 \mathrm{~g}$ i.v. twice daily for next 3-5 days.

All patients follow up was done after 1week, 1 month, 3month and 6month. Wound healing and fracture reduction was assessed by repeat $\mathrm{CT}$ at 1 month and 6month.

$80 \%$ of the patient had normal visual acuity of $6 / 6$

$20 \%$ had decreased vision ranged from 6/36-6/18. No one was blind.

All patients with orbital wall trauma managed medically or surgically showed good reduction of fractures. Wounds healed without any complication in our study. 

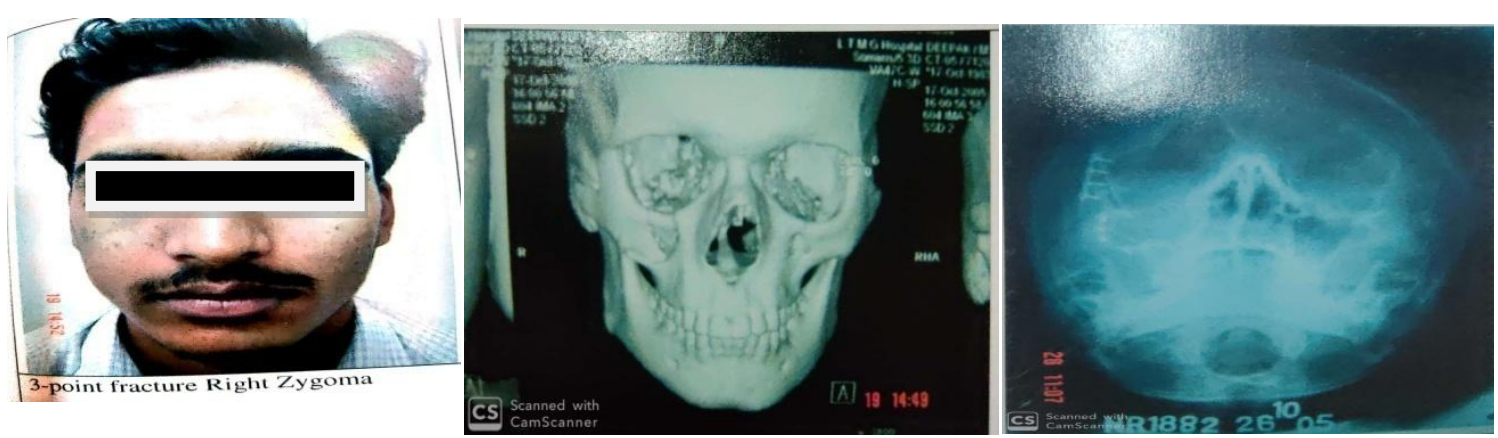

F 1-3point fracture right zygoma, F 2- 3D CT scan of 3point fracture right zygomatic complex, F 3-Waters view showing ORIF of fracture right zygomatic complex.
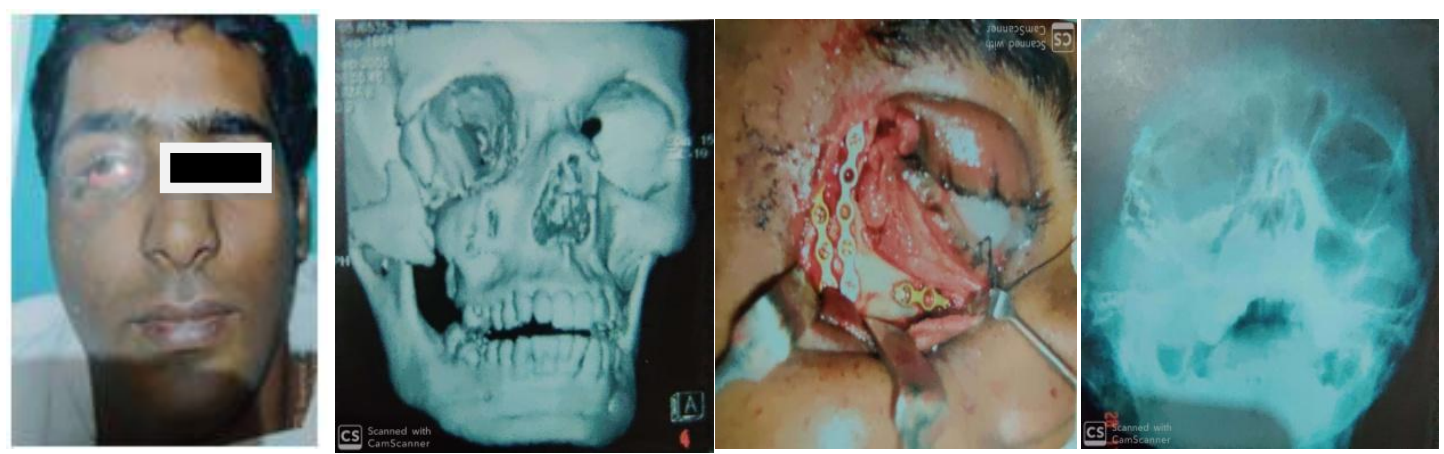

F 1-Displaced fracture of right zygoma, F 2- Grossly displaced comminuted fracture of right zygoma complex-oblique view, F 3- ORIF of right zygomatic fracture with mini-plates and screws. F 4- Waters view showing ORIF of right zygoma complex fracture.

\section{Discussion}

Road traffic accident are the most common cause of orbital and maxillofacial fractures the pattern of which is influenced by geographic area.

More than $80 \%$ of people in developing country using two wheelers and majority of them falling in productive age group 20-40 years, are prone for orbital injuries and ocular complications.

Management of such injuries involves multidisciplinary approach, ophthalmologist and other specialists. Medical and neurological stability should be ensured in all patients with ocular trauma prior to evaluation and management of ocular injuries. The capability of performing the correct diagnosis and carrying out rapid therapies effects the functional results with little aesthetic damages.

In our prospective study of 25 patients with history of road traffic accidents while driving two wheeler. Majority of cases fell in age group 2040years, with male preponderance about 19 patients were males. 24\%(6)of patient had isolated lateral wall fracture, $8 \%(2)$ had isolated medial wall fracture, $8 \%(2)$ had isolated floor fracture, lateral wall fracture combined with other wall fracture was maximum 60\%(15).

The following ocular complications were noted in our study:

All patients had periorbital ecchymosis, and subconjunctival hemorrhage.

Lid laceration was noted in 10 patients that were sutured using with 8-0 silk.

Extra ocular muscle movement restriction was noted in 8 patients. After treatment all patient regained normal extraocular muscle movement at the end of follow up.

Enophthalmos, diplopia and infraorbital anesthesia was noted in 2 patients who had severe orbital floor fracture which was corrected by surgery, orbital floor reconstruction was done using titanium mesh by transconjunctival approach after releasing of entrapped ocular muscle and fracture reduction. Displaced comminuted lateral wall (zygoma) fractures was 
managed by open reduction once periorbital swelling decreased within 10 days with interfragment wire fixation or plate and screw fixation under general anesthesia.

2 patient developed traumatic cataract after 2 months for which small incision cataract surgery with posterior chamber intraocular lens implantation was done post op vision was 6/9-6/6. 4 patients had vitreous hemorrhage which was managed medically. No other major ocular complication was noted in our study

\section{Conclusion}

In prospective study of 25 patients with history of road traffic accidents while driving two wheeler carried out for period of one year with orbital injuries. Multiple orbital wall fracture was common about $60 \%$, followed by isolated lateral wall fracture about $24 \%$.

lateral orbital wall fracture are most common in two wheeler drivers following road traffic accidents because lateral orbital wall being most prominent orbital structure susceptible to direct impact after fall from two wheeler. Multiple orbital wall fracture was common in two wheeler driver without helmet.

Multidisciplinary approach is crucial for having a predictable and successful management.

Limitation of our study was included only 25 cases, and studying orbital fracture and ocular complication only in road traffic accidents.

\section{References}

1. Herford A.S., Tandon R., Pivetti L., Cicciu M. Treatment of severe frontobasilar fractures in growing patients: A case series evaluation. chin.j traumatol.2013; 16(4): 199-203.

2. Piombino p.,Spinzia A.,Abbate V., Bonavolonta P., Orabona G.D., Califano L.Reconstruction of small orbital fractures with resorbable collagen membranes. J. Craniofac. Surg.2013;24(2):571-574.

3. Guven O.A comparative study on maxillofacial fractures in central and eastern Anatolia. A retrospective study. J craniomaxillo fac. Surg .1988;126-129.

4. Fama F.,Cicciu M.,Sindoni A., Nastrosiniscalchi E., Falzea R. Maxillofacial and concomitant serious injuries: An eight year single centre exoerience .Chin .j Traumatol.2017;20(1) :4-8.

5. Jank S., Deibl M., Strobl., Oberrauch A., Nicasi A., Missmann M.,Bodner G. Intrarater reliability in the ultrasound diagnosis of medial and lateral orbital wall fractures with a curved array transducer .J. oral maxillofac.surg.2006;64(1):68-73.

6. Manolidis S., Weeks B.H., Kirby M., Scarlett M., Hollier L. Classification and surgical management of orbital fractures: Experience with 111 orbital reconstructions. J. Craniofac. Surg. 2002;13(6):726-737.

7. Herford A.S.,Miller M., Lauritano F., Cervino G.,Signorino F., Maiorana C. The use of virtual surgical planning and navigation in the treatment of orbital trauma. Chin . J. Traumatol.2017: 20(1):913.

8. Dingman, R.O.\& Natvig, p.(1964). Surgery of Facial Fractures. Philadelphia, Toronto, London: W.B. Saunders.

9. Lang, W.; Traumatic Enophthalmos with retention of perfect acuity of vision.Trans ophthal.soc. U.K., 1889; 9:41

10. Freeman, B.S.: Direct approach to acute fractures of the zygomatic -maxillary complex and immediate prosthetic replacement of the orbital floor. Plast.Reconst Surg., 1962, 29:587

11. Tanaka N., Hayashi S., Suzuki K., Uchide N. Tomitsuka K., Hirata Y., Clinical study of maxillofacial fractures sustained during sports and games. Kokubyo Gakkai Zasshi.1992;59(3)571-577.

12. Moshy J., Mosha H.J., Lema P.A. Prevalence of maxillomandibular fractures in mainland Tanzania. East Afr.Med. j. 1996;73(3):172-175. 
13. Suzuki M., Nakamura y., Ozaki S., Yokota M., Murakami S. Repair of orbital floor fracture with modified transnasal endoscopic approach through anterior space to nasolacrimal duct .J.Craniofac. Surg.2017;28(4):998-1002. 